\title{
Heat Pump and Steam Accumulator Electrical Energy Storage System (Esheatpac System)
}

\author{
Rafael Olavarria Rodriguez-Arango,
}

Industrial Engineer, Superior Technical School Industrial Engineers (Sevilla), Sevilla University (Spain), Independent Energy Consultant

Doi:10.19044/esj.2020.v16n3p21 ～URL:http://dx.doi.org/10.19044/esj.2020.v16n3p21

\begin{abstract}
Esheatpac is an electricity storage system that combines heat pump, steam accumulator, and steam-water cycles technologies. It includes a heat pump, powered by an electric compressor, which produces saturated steam that is stored in steam accumulators as pressurized liquid water. Later, this steam produces electricity in a turbo-generator. Combination of efficiency of heat pump and Rankine cycle heat rate allows reaching efficiencies of up to $100 \%$ or higher, without requiring any auxiliary fuel. Efficiencies of up to $124.5 \%$ can be achieved by providing natural gas, with the combination of heat pumps with COP equals 2.65 and Rankine cycles with heat rate of $47 \%$. The above means that it is possible to extract from the system the same or higher amount of electricity that enters it, after remaining stored for the time required, up to almost $25 \%$ more. Esheatpac is an optimal solution for when it is required to store large amounts of electricity and medium discharge times. Today, the only storage systems that meet these conditions are pumped hydro storage (PHS) and compressed air energy storage (CAES). Compared to PHS, Estheapc presents the advantages of its better performance, a maximum of $85 \%$, and less environmental and public opposition problems, in addition to the limitations to find suitable locations. Compared to CAES, it presents the advantages of its better performance, $50 \%$ in current plants, and much lower storage capacities, on the order of seven times less, which also means a lower material investment.
\end{abstract}

Keywords: Electrical Storage, Electricity Storage, Heat Pump, Steam Accumulator, Esheatpac Electrical Energy Storage, Heat Pump And Steam Accumulator Electrical Energy Storage

\section{Introduction}

Two characteristics of electrical systems encourage the use of energy storage. First, electricity must be consumed while it is generated, so the 
electricity produced should always satisfy the existing demand. Second, the place where electricity is generated is usually far from where it is consumed, and expensive investments are required to construct transmission lines, and prevent service interruptions, or low-quality electricity supply due to overload of existing lines.

The increasing ratio of energy production from renewable sources, mainly from wind and solar power, increases the above related electricity characteristics. First, the electricity generation from these renewable resources is intermittent because it depends on ambient conditions, such as wind and sun radiation. Second, areas with high concentrations of wind or solar radiation are commonly far from locations with high electricity demand. The need and advantages of electricity storage systems increase with increased differences between offer and demand, and distances between generation and consumers. Many studies, including estimations, about future needs of electricity storage systems have been published. For example, the International Renewable Energy Agency (IRENA,2017) expects that the total electricity storage capacity will need to grow from an estimated 4.67 terawatt-hours (TWh) in 2017 to $11.89-15.72$ TWh (155-227\% higher than in 2017) by 2030, if the share of renewable energy doubles.

Electrical energy storage (EES) systems have distinctive functions (IEC, 2011): balance power generation and demand; price arbitrage; voltage and frequency control; optimize electrical grid transport and distribution; secure an improvement in the quality and manage the supply from the user's point of view. Each EES systems is suitable for one or several of the above functions.

This study presents an invention, the Esheatpac system, which is a new thermal system to store electrical energy overproduction. It can produce electricity afterwards, balance or compensate offer and demand electricity curves, and perform price arbitrage.

Nowadays, the only technologies available for high storage capacity (from 10 MW to hundreds of megawatts) and medium discharge times (hours), which are required to provide balance between production and demand curve, and price arbitrage, are: pumped hydro storage (PHS) or compressed air energy storage (IRENA,2017).

PHS are highly tested commercial installations.

In compressed air energy storage systems (Wang et al.,2017), air is compressed by electrical driven compressors and stored in pressure vessels or underground caverns. When the electricity demand increases, this stored compressed air is heated and used to operate a gas turbine to generate electricity. The air compression releases heat and, depending on the use of this released heat there are different compressed air energy storage systems (CAES). 
CAES are diabatic or conventional when the heat released during compression is dissipated to the atmosphere, and auxiliary fuel or other auxiliary heat source is required to heat the air before feeding the gas turbine. In adiabatic CAES, released thermal energy is stored and used to heat the air before feeding the gas turbine.

Nowadays, there are two diabatic CAES plants in the world: the 290 MW Huntorf Plant, in Germany (Crotogino et al.,2001); and the $110 \mathrm{MW}$ AEC Mcintosh Plant, in Arizona (Nahkamkin et al.,2007). The latter has a more advanced diabatic technology compared with the Huntorf Plant. The Mcintosh Plant adds an economizer to preheat the air from the storage cavern with exhaust gases from the gas turbine before feeding this gas turbine, so the plant efficiency is improved (advanced diabatic CAES).

There are no commercial adiabatic CAES plant in the world. However, a consortium formed by several companies is developing a demonstration plant with this technology: ADELE demonstration project (Zunft,2015).

The Esheatpac shows important advantages compared with the above related technologies, as it is explained in the following sections.

\section{Description}

The Esheatpac includes a heat pump, in which the compressor is driven by an electrical motor powered by the electrical energy from the external grid, as shown in Fig. 1. This electrical energy is introduced in the system to produce steam, with a high heat pump coefficient of performance (COP), when there is an energy overproduction, or the price is low (charge period). The produced steam is stored in steam accumulators as compressed or pressurized saturated water, and discharged as saturated steam (discharge period), when the demand of electricity is higher than the offer, or the electricity price is high. Electricity is produced in a turbo-generator or steam turbine, which operates in accordance with the Rankine water-steam cycle.

This system comprises the following main elements: heat pump, steam accumulators, turbo-generator operated by a water-steam cycle, and a watersteam condenser cooling system.

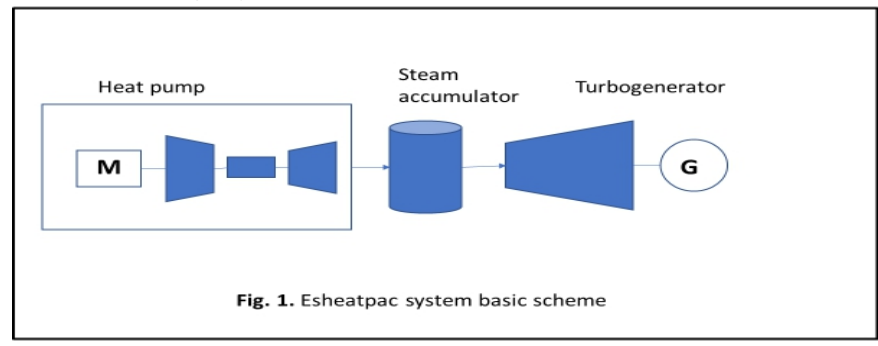

Heat Pump comprises the following main elements:

- A compressor, driven by an electrical motor, which is the element that receives the most important part of the electrical energy to be 
introduced in the system. It uses this electrical energy to compress the heat pump fluid (ammonia or other refrigerant fluid) up to the pressure required to match the required steam generator temperature.

- Heat pump and water-steam cycle have a common element, the steam generator. In the steam generator, the compressed fluid is introduced by the heat pump, and the feedwater is introduced from the watersteam cycle. Heat from the compressed fluid produces steam, heating the feedwater. This steam is stored as pressurized or compressed saturated water in steam accumulators.

- A turboexpander (or expansion turbine), that expands the heat pump fluid after the steam generation. It moves the turboexpander shaft coupled with the motor compressor shaft, reducing the required electrical power to drive the compressor, thus increasing the heat pump COP. It is possible to have one or more expansion stages, with expansion turbines operating in series. Each turbine of the expansion stage may be replaced or complemented by Joule Thompson expansion valves, where the fluid expands at constant enthalpy, without work production. Additionally, when there are several stages, evaporators to remove the heat pump fluid should be installed between stages or expansion turbines. The required energy for these evaporators will be supplied from ambient air or other waste energy source.

- Evaporator and heater. In these heat exchangers, after the last expansion stage, the heat pump fluid receives heat from ambient air or other waste heat source, until it reaches the compressor inlet temperature. The use of energy from ambient air or other waste energy sources allows the heat pump to reach high efficiency.

The heat pump fluid evaporates at temperatures lower than $0{ }^{\circ} \mathrm{C}$.

Therefore, it is also possible to use hot water from the condenser cooling system to evaporate the heat pump fluid. This hot water is cooled until the temperature is near $0{ }^{\circ} \mathrm{C}$ or until ice is produced.

This ice or cooled water is stored in well-insulated tanks. During the electricity production periods, this water may be used as cooling water in the condenser cooling system. Therefore, it can decrease the condenser vacuum pressure, increasing the Rankine cycle efficiency, and reducing the electrical and water consumption.

The steam is stored in accumulators, as pressurized saturated liquid water, then discharged as saturated steam to power a turbo-generator or a steam turbine, producing electricity.Moreover, this saturated steam may be overheated to reach superheat temperature to power the turbo-generator. It can also be reheated after the steam turbine expansion to be re-introduced in the steam turbine, increasing the Rankine cycle efficiency. These overheating or 
intermediate re-heating to reach superheat temperatures are possible by: superheating with the steam from the accumulators at temperature higher than the steam to be introduced in the steam turbine from the accumulator; reheating with the steam from the accumulators at temperature higher than the steam, to be introduced in the steam turbine after the expansion stages; using superheater or reheater boilers powered by auxiliary fuel, such as natural gas, biomass, and coal; superheating or reheating the saturated steam from the accumulators or from the expansion stages using thermal solar energy from solar receivers that can produce superheated or reheated steam, or from solar parabolic through receiver, which can also produce superheated or reheated steam. Additionally, the use of other thermal energy sources, such as geothermal energy, should also be possible.

\section{Efficiencies}

The performance of the Esheatpac system is calculated by the division between the electrical energy produced by the turbo-generator and the electrical energy of the system's inlet. This electrical energy of the system's inlet is the sum of the required energy to power the compressor motor and the energy to power the feedwater pump. The total electricity produced by the turbo-generator is calculated by the electrical energy produced in the generator outlet minus the electrical energy required to operate the condensate pump. In the Esheatpac, the heat pump performs a similar function than a boiler in a conventional thermal electric plant. Therefore, it is possible to define the efficiency of this system as the product of the heat pump efficiency (COP) and the efficiency of the water-steam cycle (Rankine cycle efficiency or heat rate) designed to produce the outlet system electrical energy, using the steam stored in the accumulators as pressurized water.

The heat pump COP is the percentage or relation between heating provided by the heat pump and electricity consumed by the heat pump.

The heat pump COP is higher than 1 (or 100\%) because the heat pump is moving heat by using energy, instead of producing heat as electrical resistance (Lee et al.,2017). A significant part of this heat is supplied by ambient air enthalpy or by a waste heat source, such as the hot water from the condenser cooling system.

For every heat pump, the heat transferred to heat sink is the sum of the heat extracted from the heat source and the energy consumed in the compressor, transmitted to the heat pump fluid, minus the energy produced in the expansion turbines, transmitted to the compressor shaft. This energy produced in the expansion turbines is the waste energy from the heat pump fluid after providing heat at the heat source, and before being conducted to the heat sink.

$$
\mathrm{QC}=\mathrm{QF}+\mathrm{WC}-\mathrm{WX} \text {, where: }
$$


$\mathrm{QC}$ is heat given to heat sink; QF is heat from heat source; WC is compressor work; and WX is expansion work.

In accordance with the COP definition (relation between provided heat and consumed electricity), the $\mathrm{COP}$ of the proposed electrical storage system is:

$$
\mathrm{COP}=\mathrm{QC} /(\mathrm{WC}-\mathrm{WX})=(\mathrm{QF}+\mathrm{WC}-\mathrm{WX}) /(\mathrm{WC}-\mathrm{WX})>1
$$

QC is the heat given by compressed fluid to the steam generator to produce saturated steam, which is stored in accumulators as pressurized or compressed liquid water.

QF is the heat from ambient air, or from a waste heat source, or from the condenser cooling water. It is used to evaporate and preheat liquid, or partially evaporate the heat pump fluid, at temperature lower than ambient air temperature after expansion stages.

The more heat can be extracted from the ambient air, or from other waste source, the higher the heat pump COP.

From a theoretical point of view, the heat pump COP, operating as a heat source, depends on heat source and heat sink temperatures, in accordance with the following equation:

$$
\mathrm{COP}=1 /(1-(\mathrm{T} 2 / \mathrm{T} 1)), \text { where: }
$$

T2: Heat source absolute temperature.

T1: Heat sink absolute temperature.

This COP represents the maximum theoretical value, which is 14.65 when the external temperature is $0{ }^{\circ} \mathrm{C}$, and the internal temperature is $20{ }^{\circ} \mathrm{C}$. In other words, it is possible to generate 14.65 thermal kilowatts for each electrical kilowatt consumed, from a theoretical point of view.

The heat sink temperature proposed by the Esheatpac system depends on feedwater pressure, the temperature (saturated temperature) required to generate steam at elevated pressure, and the temperature to produce electricity at reasonable Rankine cycle efficiency. This temperature is higher than the above referenced $20^{\circ} \mathrm{C}$.

In a theoretical calculation, it is possible to achieve a heat pump COP around 3, generating steam at around 100 bars. This steam can operate with a high Rankine cycle efficiency.

The Rankine cycle is a thermodynamic cycle used to produce electricity in thermal electric plants.

As part of the Rankine cycle in this Esheatpac system, after being superheated (or not), saturated steam flowing out of the steam accumulators is conducted to a steam turbine, where it expands, producing mechanical work by rotating the shaft coupled to the generator shaft to produce the outlet system electricity. It is possible to have one or several expansion stages and, after each expansion stages, steam may be reheated or not. 
After the last expansion stage, the outlet steam from the steam turbine is discharged into a condenser, where it condensates. This condensed water is pumped by the condensate pump and feedwater pump until it reaches the pressure required by the heat pump steam generator to match the pressure required by the pressurized saturated liquid water to be stored in the accumulators.

This Rankine water-steam cycle has some distinctive characteristics concerning pumping operations, because the heat pump cycle and the Rankine cycle operate at different periods.

- Condensate pumps operate during discharge periods, when the steam turbine is in operation. These pumps conduct water from the outlet condenser to the condensate storage tank, where the condensate water is stored at atmospheric pressure until an additional charge period starts. As a result, the auxiliary electrical consumption of these condensate pumps must be reduced from the gross power to match the steam turbine net power.

- Feedwater pumps operate during charge periods, when the steam turbine is not in operation. Therefore, these pumps are driven by motors powered with electrical energy from an external grid, and not by electrical motors powered by the energy generated by the turbo-generator, nor using turbopumps, as it is usual in conventional electrical generation plants.

As a result, the electrical energy of the entire inlet system is the sum of the electrical energy which powers the heat pump compressor and the electrical energy which powers the feedwater pump motor. The electrical energy of the entire outlet system is the electrical energy generated by the turbo-generator at the outlet generator terminal minus the required electrical energy to power the condensate pump motor. 

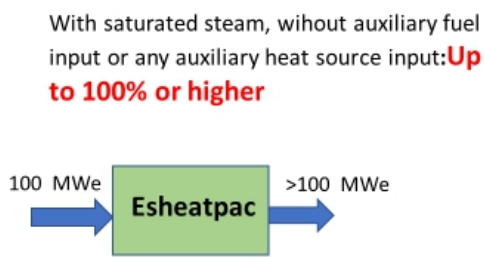

Rankine cycle efficiency > 38\%

COP Heat Pump $>2,65$
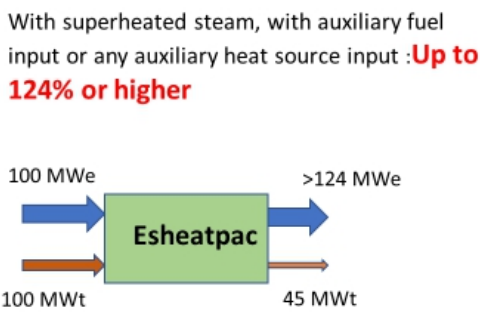

Rankine cycle efficiency: $>47 \%$

COP Heat Pump : > 2,65

Fig. 2. Esheatpac system performances

The efficiency of the saturated steam from the Rankine cycle, or the heat rate, is approximately $38 \%$. Subcritical superheated and reheated Rankine cycle efficiency is higher than $47 \%$. Both cycles efficiency does not include the boiler or a make-up heat system, because this function is performed by the heat pump.

As a result, the efficiency of the Esheatpac system is higher than $141 \%$ when the heat pump COP is 3 and the Rankine cycle efficiency is $47 \%$. In other words, the inlet electrical energy is multiplied by 1.41 to match the outlet electrical energy.

From a practical point of view, considering the requirements of ammonia as a fluid, the maximum foreseen heat pump efficiency is around 2.65, including losses.

Therefore, efficiency reaches up to $100,7 \%$ when the heat pump COP and saturated steam Rankine cycle efficiencies are above the referenced values of 2.65 and $38 \%$. When the superheated and intermediate reheated steam are present in the Rankine cycle, the cycle efficiency is higher than the referenced value of $47 \%$. Thus, the efficiency of the Esheatpac reaches $124,5 \%$, which means that the electrical energy of the outlet system is higher than the inlet stored electrical energy.

\section{Practical Case}

Fig. 3 shows a heat pump cycle and a Rankine cycle whose combination is an EES system with a heat pump and steam accumulators (Esheatpac).

It is noted that this practical case does not have to correspond to a design with maximum or optimum performance or profitability.

These optimizations must be made for each project, and will depend on numerous factors including: the purchase and sale prices of electric power; 
the hours expected of charge and discharge; the site and environmental conditions; the specific requirements and conditions of each project; the cost correlation between the different equipment and elements of the project that may exist at any time and in any place.

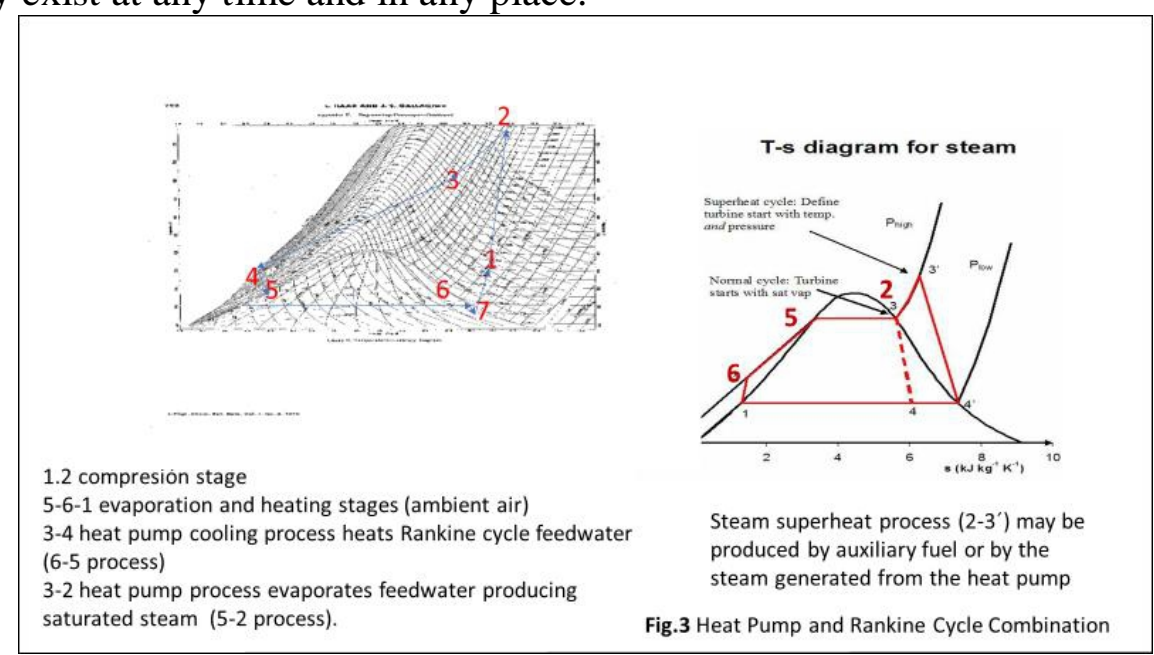

Fig.3 Heat Pump and Rankine Cycle Combination

The input conditions of this practical case are the following:

- Ambient temperature: $27^{\circ} \mathrm{C}$.

- Heat pump fluid: Ammonia.

- Ambient air for evaporation and heating stage.

The first stage of the charge period is the compression of the fluid from the heat pump (1-2 stage). This fluid coming from the storage tank will be at a temperature close and lower than the air temperature from the environment used for its evaporation and heating. This compression will be carried out in several stages up to 110 bar.

After leaving the compressor, the heat pump fluid enters the steam generator (2-3) to generate saturated steam from the feed water of the Rankine cycle. Saturated steam at pressure higher than 100 bar is generated, feeding the accumulators.

This feed water is previously heated (6-5 stage of Rankine cycle) by the heat from the pump fluid that leaves the steam generator (3-4).

To generate saturated steam, various stages of compression and generation can be provided to increase cycle efficiency.

The ammonia, after this last heating stage, is at a pressure slightly lower than 110 bar and is introduced in the first expansion turbine (4-5). It expands to an intermediate pressure, reaching a lower temperature than the ambient, producing work that is used to partially drive the compressor shaft. 
Later, this ammonia, at intermediate pressure, is evaporated by using ambient air and undergoing a subsequent expansion to a pressure close to atmospheric and a temperature below $-30{ }^{\circ} \mathrm{C}$.

Finally, the ammonia is heated by the ambient air to the initial conditions (point 1), which is at atmospheric pressure and temperature lower than the ambient temperature.

In this case, two stages of expansion have been designed. Although there could be more than two stages, which would improve efficiency, that would also make the system more expensive.

In the discharge periods, the steam stored in the accumulators, such as pressurized water at saturation temperature, is discharged by flash evaporation as saturated steam at $100 \mathrm{bar}$, which is the value selected as the inlet pressure of the steam turbine. The heat required for this process comes from the enthalpy difference in the pressurized water at storage conditions and discharge conditions.

The output or vacuum pressure of the turbine must be the minimum possible to improve the performance of the Rankine cycle, but respecting the requirements of maximum humidity for the saturated steam that leaves the turbine to establish the supplier. This minimum pressure, in addition to the requirements of the supplier, will be limited by the environmental conditions of the site and water availability. In the case of the proposed system, it will be limited by the cooling capacity that can be taken from evaporators and heaters. In this practical case, a vacuum pressure of 0,025 bar has been set.

The results that were obtained are:

- Compression power: 134,5 MWe.

- Expansion Power: 28 MWe.

- Thermal power supplied to the Rankine cycle: 268 MWt.

- Steam turbine power: 96,5 MWe.

- Feedwater pump power: $2.5 \mathrm{MWe}$.

The EES system efficiency is the ratio of the outgoing electric power produced by the incoming electric power divided by the same incoming electric power.

The outgoing electrical power $(96,5 \mathrm{MWe})$ is generated in the turbogenerator, except to drive the condensate pumps.

The incoming electric power (109 MWe) is the one that feeds the compressor, which will be the power needed for its operation except for the one provided by the expansion turbines, plus the one that feeds the feedwater pump.

The quotient between the two previous powers gives us a system efficiency of $88,5 \%$, including losses. 
This efficiency can also be defined as the product of the heat pump COP by the efficiency or heat rate of the water-steam Rankine cycle.

Heat pump efficiency is the ratio between the heating provided and the electricity consumed. The heating provided is the sum of the energy supplied to the generator plus the energy supplied to the feed water heater, minus the losses $(268 \mathrm{MWt})$. The consumed electrical power is the one supplied to the compressor and the feed water pump, except for the one provided by the expansion turbines (109 MWe). The quotient between both gives us a COP of 2.46 .

The heat rate of the Rankine cycle is defined as the generated electrical power (96,5 MWe) divided by the thermal power supplied to the cycle. In our case, the resulting heat rate of the cycle was $36 \%$. The product of the COP and Rankine cycle heat rate gives an EES system efficiency of $88,5 \%$, which is identical to the one previously calculated, as expected.

It should be noted that the power generated by the steam turbine from the steam stored in the accumulators corresponds to a situation in which the hours of charge are equal to those of discharge. If they were different, the power of the steam turbine would be different. For instance, if the hours of discharge were half the hours of charge, the power of the steam turbine would practically double. The above does not affect the calculated EES system performances, except for the small differences that may exist in the efficiencies of steam turbines of different sizes.

Assuming we work with a superheated steam cycle, the heat rate can reach values of $45 \%$ or higher, and the efficiency of the Esheatpac system could rise to $110,7 \%$.

This practical case allows numerous variations that can improve the storage efficiency. However, these improvements may imply an increased project cost that would not compensate their implementation. Consequently, each variation must be analysed from the point of view of profitability, within the context of each project.

Some of the possibilities to improve the performance or the heat pump COP, currently or in the future, are:

- Optimize the heat pump cycle, optimizing the pressure range in evaporators and heaters.

- Increase compression, generation, and expansion stages.

- Reduce temperature differences or use an approach in which evaporators and heaters operate at close temperature.

- Improve compressor and expansion turbine efficiencies.

- Decrease losses in evaporators and heaters.

- Use other cooling fluids that improve the performance of heat pumps (Kontomaris, 2013). 
From a practical point of view, for this application and using ammonia, it is estimated that COP can reach up to 2.65 or higher.

Moreover, the storage system with accumulators allows improvements that can lead to an increase in the Esheatpac system efficiencies, such as:

- Reduction of accumulator losses, increasing their isolation.

- Optimization of the charge and discharge pressures, and reduction of the differences between both pressures. However, the reduction of the differences between these pressures can imply significant increases in storage capacities, and, consequently, in the required investments.

Finally, the Rankine cycle allows significant improvements compared to the practical case of reference.

Modern steam turbines, normally for high electrical pressures, allow isentropic efficiencies in the steam turbine to reach 94\%, and the Rankine cycle heat rates or efficiency to reach $47 \%$ and higher.

For a COP of 2.65 and heat rates of $47 \%$, an efficiency of $124,55 \%$ is obtained.

\section{Comparison with other technologies}

The Esheatpac system has important advantages if compared with other competitors with high storage capacity, from ten to hundreds of megawatts, and average discharge periods of several hours, which are the systems required to regulate the offer and demand electricity curves (IRENA, 2017).

Currently, these competitor systems are PHS and CAES (Hadjispasscalis et al.,2009 and Chen et al., 2009).

PHS plants have been well tested. There are many plants around the world in commercial operation. Nowadays, these systems have an efficiency between $76 \%$ and $85 \%$, lower than Esheatpac. They require large land areas in adequate natural sites, which are difficult to locate. For their construction, they require upper and lower water reservoirs and dams, producing large environmental impacts and, therefore, a negative public opinion.

Additionally, CAES systems have very important disadvantages compared with the Esheatpac electrical energy storage system using heat pumps and steam accumulators:

- Advanced diabatic CAES systems, with heat recovery systems (similar AES Mcintosh Plant) have net efficiencies of around $50 \%$ to convert inlet electrical energy to outlet electrical energy. The foreseen efficiency for the ADELE project (adiabatic CAES) is 70\%. Although, these efficiencies may improve in the future, owing to process and equipment improvements, they are not likely to match the Esheatpac's high efficiency. The main reason is that these high efficiencies are achieved by the contribution of heat from the ambient air, or other 
residual source of heat, during the evaporation and heating stages of the heat pump cycle.

- CAES systems store compressed gas (air) and Esheatpac stores pressurized liquid in accumulators. The density of the liquid is much higher than the compressed air density. Therefore, required storage specific capacity $\left(\mathrm{m}^{3} / \mathrm{Kg}\right)$ are much lower, at similar pressures.

- CAES systems only store thermal energy as sensible heat, because phase change in the air does not occur. Ammonia, or other refrigerant fluid, and water-steam phase change are produced during in the Esheatpac storage process. These phase changes imply that thermal energy is stored as sensible and latent heat, and not only as sensible heat, so the proposed storage specific heat capacity $(\mathrm{Kj} / \mathrm{kg})$ is much higher.

Because of the above indicated storage issues, densities differences, and phase changes, the storage volume required by the Esheatpac is much lower than the storage volume required by CAES systems, considering the same thermal capacity of storage.

The electrical energy storage AEC Mcistosh plant, with advanced adiabatic design, $110 \mathrm{MWe}$, and 26 hours of discharge capacity, have a storage capacity of $550.000 \mathrm{~m}^{3}$, at 76 bar. It is in a salt mine of geological formation (US Department of Energy). From an economical point of view, these high storage capacity requirements do not allow the use of compressed air storage tanks or accumulators, and CAES storage solutions based on geological formations are required.

Advanced diabatic CAES plant electrical energy storage capacity requirement is estimated as more than seven times higher than the required capacity of a heat pump and steam accumulator electrical energy storage plant with the same electrical power and discharge times (hours). If compared with an adiabatic CAES plant, the above difference would be higher.

As a result, storage capacities requirements and efficiencies improvements imply very important advantages of the Esheatpac system when compared with any PHS or CAES system.

Electrical systems that require high storage capacity (between $10 \mathrm{MW}$ up to hundreds MW) and medium discharge periods (hours) increase the importance of the mentioned advantages.

Therefore, Esheatpac may be especially interesting where isolated or interconnected electrical systems require a balance between offer and demand of electricity or a price arbitrage. Nevertheless, this electrical storage system can be very useful on large industrial factories with very high electrical demand (e.g. chemical factories, and steel and non-ferrous metal industries), 
where this system may reduce the costs by buying electricity during off-peak hours, when the electricity price is lower.

\section{Equipment and materials}

Similar equipment required to design the Esheatpac system exists in industrial factories or plants, with similar fluids and similar, or superior, process conditions (chemical factories, oil refineries, or electrical power plant).

Basically, the main type of equipment required are compressors, expansion turbines, steam generators or heat exchangers, steam turbines, pumps, pressure vessel, and tanks.

Several compressors suppliers offer compressors capable of compressing ammonia or similar fluids to the conditions required by the Esheatpac system, around 100 bar or higher.

Additionally, expansion turbines or turbo expanders as required by Esheatpac exist in the market, operating at the same conditions: liquid, liquid with partial evaporation, saturated, or superheated fluids turbo expanders, at around 100 bar or higher.

Other required equipment (steam turbines, heat exchangers, or pumps) are also commercial products.

It is especially important to optimize the design and properly chose the type of steam accumulators used by the system, as it significantly impacts the total project cost.

Between 20.000 and $45.000 \mathrm{~m}^{3}$ of steam accumulator capacity are required, depending on the design range of the working pressure, to produce $100 \mathrm{MWe}$ for 10 hours (1000 MWe), working at maximum efficiency, with steam from accumulators.

Steam accumulators are a well-advanced technology with well-tested experience for thermosolar plants, industrial applications, and steam demand regulations.

There are several steam accumulators options:

- Carbon steel tanks, with capacities between $150-700 \mathrm{~m}^{3}$ or higher, using special transports.

- Large diameter and high strength pipe racks; or buried pipe.

- Prestressed concrete tanks, with capacities up to $40.000 \mathrm{~m}^{3}$ or higher.

- Underground fossil water reservoir.

Carbon steel tanks or pipe racks are preferred options up to $10.000 \mathrm{~m}^{3}$. Large carbon steel tanks (if special transport to site are possible); or prestressed concrete tank; or high resistance and diameter pipes (X80/X90) are preferred when high capacities are required. 


\section{Conclusion}

The Esheatpac system is an electrical energy storage system that combines heat pumps, steam accumulators and water-steam cycles technologies.

Combination of heat pump efficiency (COP $>2)$ and turbo-generator (Rankine cycle) efficiency match global efficiencies of $100 \%$ or higher, operating with saturated steam; or $120 \%$ or higher, operating with superheated steam.

Overall, the Esheatpac system comprises:

- A heat pump with a compressor driven by an electrical motor fed by external electrical energy. This energy is introduced in the system to be stored as compressed saturated water, which can generate steam with a high efficiency (heat pump COP) when there is excessive electricity production, or its price is low.

- This generated steam is stored in accumulators as pressurized saturated water and discharged to produce electricity when electricity demand is higher than the offer or the price is high.

Nowadays, EES are essential to optimize isolated or interconnected electrical systems.

EES has several functions, including time shifting, to match the supply and demand of electricity, and price arbitrage.

These functions require systems with high storage capacity (between $10 \mathrm{MW}$ and hundreds of MW) and medium discharge times (hours).

Current EES systems matching these requirements are PHS and CAES. PHS is a mature technology, highly tested, with efficiencies between $76 \%$ and $85 \%$. The major drawback of PHS are the large amount of land required in natural spaces, with important environmental impacts and public opposition.

Today, CAES plants reach efficiencies of around 50\%, whereas the ADELE demonstration project (adiabatic CAES) foresees a 70\% efficiency.

CAES require large storage capacities, more than seven times of what is required by the Esheatpac. Therefore, natural caverns or salt mines are required.

Lower required storage capacities, which given the significant percentage that the storage elements have in the total investment, also mean lower material investment, and higher efficiencies, shows the competitive advantages of Esheatpac systems compared with CAES systems.

Higher efficiency and lower environmental and public opposition problems shows competitive advantages of Esheatpac compared with PHS systems.

Every equipment and material required to construct the Esheatpac system are commercially available. 


\section{References:}

1. Chen, H. Cong, T. N. Yang, W. Tang, C. Li, Y. Ding, Y. (2009). Progress in electrical energy storage system: A critical review. Progress in natural Science, vol 19, Issue 3, March 2009, Pages 291312.

2. Crotogino, F. Scharf, R. Mohmeyer, K. (2001). Huntorf CAES: More than 20 years of successful operation. Proceedings of the spring 2001 meeting, Orlando, Fla, USA, April 2001.

3. Hadjipasscalis, J. Poullikkas, A. Efthimou, V (2009). Overview of current and future energy storage technologies for electrical power applications. Renewable and Sustainable Energy Reviews, Vol 13, issue 6-7, August-September 2009, Pages 1513-152.

4. IEC, International Electrochemical Commission (2011). White Paper Electrical Energy Storage. Retrieved from http://www.iec.ch/whitepaper/energystorage.

5. IRENA, International Renewable Energy Agency (2017). Electricity storage and renewables: Cost and markets to 2030. International Renewable Energy Agency, Abu Dhabi.

6. Kontomaris, K (2013). Low GWP Working fluid for high temperature heat pumps: DR2. European Heat Pump Summit, Nuremberg, October 15, 2013.

7. Lee, B. Wang, E. Lee, G. Ra, H. Hyuncho, J. Baik, Y. Lee, Y (2017). Performance improvement of heat pump for generation steam. Proceedings of the Asia Conference on thermal sciences 2017, $1^{\text {st }}$ ACTS, March 26-30, 2017. ACTS-P00322.

8. Nakhamkin, M. Chirovolu, M (2007). Available Compressed Air Energy Storage (CAES) Plant Concepts. http://espcinc.com/library/Powergen_2007_paper.

9. US Department of Energy. DOE Global Energy Database. Retrieved from http://www.sandia.gob/ess/database.

10. Wang, J. Lu, K. Ma, L. Wang, J. Dooner, M. Miao, S. Li, J. \& Wang D (2017). Overview of compressed air energy storage and technology development. Energies 10(7). 991.

11. Zunft, S. (2015). Adiabtic CAES: The ADELE-ING project.

12. SCCER Heat\&Electricity Storage Symposium, PSI, Villigen $(\mathrm{CH})$, May 5,2015

13. http://www.sciencedirect.com/science/article/pil/S100200710800381 $\mathrm{X}$. 